\title{
Impact of Oncotype DX testing on ER+ breast cancer treatment and survival in the first decade of use
}

\author{
Evelien Schaafsma ${ }^{1 \dagger}$, Baoyi Zhang $^{2 \dagger}$, Merit Schaafsma ${ }^{3}$, Chun-Yip Tong ${ }^{4}$, Lanjing Zhang ${ }^{5,6}$ and Chao Cheng ${ }^{4,7,8,9^{*}}$ (D)
}

\begin{abstract}
Background: The Oncotype DX breast recurrence score has been introduced more than a decade ago to aid physicians in determining the need for systemic adjuvant chemotherapy in patients with early-stage, estrogen receptor (ER)+, lymph node-negative breast cancer.

Methods: In this study, we utilized data from The Surveillance, Epidemiology, and End Results (SEER) Program to investigate temporal trends in Oncotype DX usage among US breast cancer patients in the first decade after the introduction of the Oncotype DX assay.

Results: We found that the use of Oncotype DX has steadily increased in the first decade of use and that this increase is associated with a decreased usage of chemotherapy. Patients who utilized the Oncotype DX test tended to have improved survival compared to patients who did not use the assay even after adjusting for clinical variables associated with prognosis. In addition, chemotherapy usage in patients with high-risk scores is associated with significantly longer overall and breast cancer-specific survival compared to high-risk patients who did not receive chemotherapy. On the contrary, patients with low-risk scores who were treated with chemotherapy tended to have shorter overall survival compared to low-risk patients who forwent chemotherapy.

Conclusion: We have provided a comprehensive temporal overview of the use of Oncotype DX in breast cancer patients in the first decade after Oncotype DX was introduced. Our results suggest that the use of Oncotype DX is increasing in ER+ breast cancer and that the Oncotype DX test results provide valuable information for patient treatment and prognosis.
\end{abstract}

Keywords: Oncotype DX, Breast cancer, Prognosis, Adjuvant chemotherapy

\section{Background}

Breast cancer is the most common type of cancer in women in the world and the second most common cancer overall [1]. The incidence of breast cancer has been increasing by $3.1 \%$ every year and this trend is expected

\footnotetext{
* Correspondence: chao.cheng@bcm.edu

'Evelien Schaafsma and Baoyi Zhang contributed equally to this work.

${ }^{4}$ Department of Medicine, Baylor College of Medicine, Houston, TX 77030, USA

${ }^{7}$ Dan L Duncan Comprehensive Cancer Center, Baylor College of Medicine, Houston, TX 77030, USA

Full list of author information is available at the end of the article
}

to continue [2]. Approximately $70 \%$ of all breast cancer patients presents with ER+ human epidermal growth factor 2 (HER2)- breast cancer [3, 4], which has a 5-year overall survival rate of $>94 \%$ [5]. This high survival rate is partially due to the low rate of breast cancer recurrence following treatment of adjuvant chemotherapy in ER+HER2-, node-negative breast cancer after surgical resection [6-8]. However, since only $\sim 15 \%$ of ER+HER2 - breast cancer patients would experience recurrence at 5 years in the absence of adjuvant chemotherapy [8], $85 \%$ of patients may be exposed to chemotherapy

(c) The Author(s). 2021 Open Access This article is licensed under a Creative Commons Attribution 4.0 International License, which permits use, sharing, adaptation, distribution and reproduction in any medium or format, as long as you give appropriate credit to the original author(s) and the source, provide a link to the Creative Commons licence, and indicate if changes were made. The images or other third party material in this article are included in the article's Creative Commons licence, unless indicated otherwise in a credit line to the material. If material is not included in the article's Creative Commons licence and your intended use is not permitted by statutory regulation or exceeds the permitted use, you will need to obtain permission directly from the copyright holder. To view a copy of this licence, visit http://creativecommons.org/licenses/by/4.0/ The Creative Commons Public Domain Dedication waiver (http://creativecommons.org/publicdomain/zero/1.0/) applies to the data made available in this article, unless otherwise stated in a credit line to the data. 
toxicity with minimal clinical benefit. To decrease the overuse of adjuvant chemotherapy, patient stratification based on breast cancer recurrence risk has become an effective strategy to aid in individualized breast cancer treatment $[9,10]$.

The Oncotype DX assay has been used widely to predict the recurrence risk of breast cancer after surgical resection in ER+HER2- breast cancer [11]. Based on the expression levels of 21 genes, the test calculates a risk recurrence score from 0 to 100 that stratifies patients into three groups: high- $(\geq 31)$, intermediate- (18-30), and low-risk (0-17) of recurrence. The Oncotype DX test has been endorsed by the American Society of Clinical Oncology (ASCO) and the National Comprehensive Cancer Network (NCCN) [11, 12]. The NCCN recommends the use of Oncotype DX for ER+HER2-, earlystage (T1 or T2), lymph node-negative (pN0) breast cancer. Based on the RxPONDER trial (NCT01272037) [13, 14], patients with ER+HER2-, early-stage, lymph nodepositive $(\mathrm{pN}+)$ breast cancer also derive benefit from the Oncotype Dx assay. Since the formal validation of the Oncotype DX assay in 2004 [9], the uptake of testing has increased each year [15].

A number of studies have evaluated chemotherapy usage after Oncotype DX test results [16]. Consistently, patients with low-risk Oncotype DX scores do not benefit from the additional chemotherapy and are consequently rarely treated with chemotherapy [16-19]. On the contrary, the usage of chemotherapy is reported highest among patients with an Oncotype DX score $\geq 31$ (high risk of recurrence) compared to lower Oncotype DX scores [16, 19-21]. High-risk patients identified by the Oncotype DX assay are more likely to benefit from chemotherapy than low-risk patients in both neoadjuvant and adjuvant treatment regimens [22]. The benefit of chemotherapy in the intermediate-risk group is less clear. A large randomized trial (TAILORx) suggested that disease-free and overall survival are similar between intermediate-risk patients randomly stratified into no adjuvant treatment or adjuvant chemotherapy [23]. Other factors that are considered when deciding if adjuvant chemotherapy is recommended include the size of the tumor, lymph node status, differentiation, time period of treatment, and patient age [24, 25]. For example, approximately $90 \%$ of patients aged $<40$ with a high recurrence score were treated with chemotherapy, whereas only $50 \%$ in the elderly ( $>80$ years) population was treated with chemotherapy [26].

In this study, we performed a systematic analysis on the Surveillance, Epidemiology, and End Results (SEER) breast cancer dataset [27]. We aimed to provide a comprehensive overview of Oncotype DX testing rates and how these are related to different clinical variables. Although prognostic relationships between Oncotype DX scores and patient survival have been studied, we wanted to provide a year-by-year overview of prognostic associations while also adjusting for important clinical variables to evaluate if prognostic trends could be observed. These analyses were conducted with the ultimate goal of providing useful information to refine the guidelines for directing Oncotype DX tests.

\section{Materials and methods}

\section{SEER-Oncotype DX database}

The SEER-Oncotype DX data for breast cancer patients diagnosed between 2004 and 2015 were used in this analysis. These data were generated by linking Oncotype DX test results with invasive breast cancer cases from 17 SEER registries [27]. The SEER database provides patient clinical information including year of diagnosis ("Year.of.diagnosis"), age ("Age.at.diagnosis"), race ("Race.ethnicity"), tumor type ("Breast.Subtype.2010"), grade ("Grade"), stage ("Breast.Adjusted.AJCC.6 ${ }^{\text {th }}$.Stage.1988.2015"), and ER status ("ER.Status.Recode.Breast.Cancer.1990"). Breast cancer-specific survival (BCSS) ("SEER.cause.specific.death.classification") and overall survival (OS) ("Vital.status.recode.study.cutoff.used") were also provided. For patients who underwent Oncotype DX testing, test results were provided as continuous recurrence-risk scores ("Oncotype.DX.Breast.Recurrence.Score") and according to risk categories provided by SEER: high (risk score $>30$ ), intermediate (risk score 18-30), and low-risk (risk score < 18) ("Oncotype.DX.RS.group.RS.18.RS.18.30.RS.30"). In this study, we focused solely on ER+ breast cancer patients aged 35 to 80 years at the time of diagnosis. Of note, patients with ER+HER2+ breast cancer were patients whose tumors were HER2+ according to SEER data but the Oncotype DX report indicated HER2- status per RTPCR. Consequently, the ER+HER2+ patient group described in this study is a subgroup of those with ER+ HER2 + breast cancer and does not resemble all patients with ER+HER2+ breast cancer. Patients with precancerous disease (stage $=0$ ) were excluded from the study. The final data contained a total of 375,350 unique patient IDs. Among these patients, 89,255 patients (24\%) underwent Oncotype DX testing with 6414 (7\%), 31,302 (25\%), and 51,539 (58\%) high-, intermediate-, and lowrisk test results, respectively. All exact SEER variable names have been provided in quotation marks above.

\section{Survival analysis}

Survival analyses were performed using the $\mathrm{R}$ survival package (version 3.2-7). Log-rank tests were performed to evaluate overall or breast cancer-specific survival probabilities between two groups, using the "survdiff" function. Univariate Cox proportional hazards regression was performed on continuous Oncotype DX scores 
using the "coxph" function. Multivariate Cox proportional hazards regression was performed similarly while adjusting for age, tumor stage, lymph node status, and breast cancer subtype for years 2010-2015. Hazard ratios were extracted from both univariate and multivariate models for Oncotype DX scores. Kaplan-Meier plots were generated using the "survfit" function from the $\mathrm{R}$ survival package.

\section{Statistical analyses}

All analyses were performed in $\mathrm{R}$ (version 3.6.2). Descriptive statistics were used to summarize the SEER data according to patient and tumor characteristics. Ttest and chi-squared test was performed to determine the differences in continuous and categorical patient characteristics between Oncotype DX usage (user vs. non-user). All tests were two-sided if applicable and statistical significance was assessed using an alpha of 0.05 .

\section{Results}

\section{Application of Oncotype DX test in ER+ breast cancer patients}

Utilizing the SEER database, we first defined clinical differences among 375,350 breast cancer patients who were stratified based on the usage of Oncotype DX (users vs. non-users) (Table 1). Multivariable analysis indicated that patients with lower tumor stages, lower grade, negative lymph node status, negative HER2 status, and of

Table 1 Patient characteristics of the SEER-Oncotype cohort. $P$ values were calculated by t-test and chi-squared test for continuous and categorical characteristics, respectively

\begin{tabular}{|c|c|c|c|}
\hline & Users $(n=89,255)$ & Non-users $(n=286,095)$ & $P$ value \\
\hline Mean age (SD) & $57.8(10.0)$ & $59.5(11.5)$ & $<1 \mathrm{e}-314$ \\
\hline Stage (\%) & & & $<1 \mathrm{e}-314$ \\
\hline । & $58,512(65.6)$ & $132,415(46.3)$ & \\
\hline$\|$ & $29,494(33.0)$ & $97,347(34.0)$ & \\
\hline III & $1104(1.2)$ & $40,265(14.1)$ & \\
\hline IV & $145(0.2)$ & $16,068(5.6)$ & \\
\hline Grade (\%) & & & $<1 \mathrm{e}-314$ \\
\hline । & $70,648(26.1)$ & $25,215(28.9)$ & \\
\hline$\|$ & $126,526(46.8)$ & $47,690(54.7)$ & \\
\hline III & $73,256(27.1)$ & $14,338(16.4)$ & \\
\hline Lymph node (\%) & & & $<1 \mathrm{e}-314$ \\
\hline Negative & $76,401(85.6)$ & $174,024(60.8)$ & \\
\hline Positive & $12,848(14.4)$ & $109,956(38.4)$ & \\
\hline Unknown & $6(0.0)$ & $2115(0.8)$ & \\
\hline HER2 (\%) ${ }^{a}$ & & & $<1 \mathrm{e}-314$ \\
\hline Negative & $62,115(95.3)$ & $112,187(78.2)$ & \\
\hline Positive & $1199(1.8)$ & $24,469(17.1)$ & \\
\hline Unknown & $1889(2.9)$ & $6850(4.7)$ & \\
\hline Race (\%) & & & 3.E-71 \\
\hline White & $73,722(82.6)$ & $230,090(80.4)$ & \\
\hline Black & $7129(8.0)$ & $69,307(10.0)$ & \\
\hline Asian & $6837(7.7)$ & $22,592(7.9)$ & \\
\hline Other & $1293(1.4)$ & $3962(1.4)$ & \\
\hline Unknown & $274(0.3)$ & $851(0.3)$ & \\
\hline Chemotherapy (\%) & & & $<1 e-314$ \\
\hline No/unknown & $67,853(77.7)$ & $159,334(55.7)$ & \\
\hline Yes & $19,948(22.3)$ & $126,761(44.3)$ & \\
\hline Radiation therapy (\%) & & & 7.E-313 \\
\hline No/unknown & $34,763(38.9)$ & $132,040(46.2)$ & \\
\hline Yes & $54,492(61.1)$ & $154,055(53.8)$ & \\
\hline
\end{tabular}

${ }^{a}$ Since only samples after 2009 contained breast cancer subtype information, the displayed percentages include patients from 2010 to 2015 only 
Caucasian descent were all significantly more likely to be an Oncotype DX user compared to non-users (Table 1). Discrepancies in Oncotype DX testing rate between different races have indeed been observed in previous studies $[20,28]$. Oncotype DX users were also less likely to have received chemotherapy, but they were more likely to have received radiation therapy compared to nonOncotype DX users.

The usage of Oncotype DX steadily increased over the last decade with $34 \%$ of all ER+ breast cancers undergoing testing in 2015 (Fig. 1A). The percentage of patients with ER+HER2+ breast cancer who underwent testing remained relatively stable around $3 \%$ of all ER+HER2+ breast cancer patients. Notably, ER+HER2+ patients in this cohort were classified as HER2+ by SEER but showed HER2 negativity according to Oncotype DX HER2 testing. Thus, this patient group likely resembles a subset of ER+HER2+ patients. Consequently, our reported testing rate of $3 \%$ is likely an underestimation of the true testing rate in ER+HER2+ breast cancer. Most Oncotype DX users had early-stage disease (Fig. 1B), consistent with current Oncotype DX guidelines [14]. However, a slightly increasing trend in Oncotype DX testing was observed in stage III breast cancer patients, starting from $0 \%$ in 2004 to $6 \%$ in 2015 (Fig. 1B). Tumors with lower tumor (grade I and II) were more likely to use Oncotype DX testing compared to high grade (grade III) (Additional file 1 - Figure 1A). Oncotype DX usage increased over time in both lymph node-negative and positive breast cancer (Fig. 1C). The usage of Oncotype DX in lymph node-negative breast cancer patients was more than twice the usage of Oncotype DX in lymph node-positive breast cancer (Fig. 1C). Lastly, differences in Oncotype DX usage were observed between different age groups. Older breast cancer patients (6080 years) were least likely to utilize Oncotype DX throughout the entire study period (Fig. 1D), whereas middle-aged patients (45-60 years) were most likely to utilize Oncotype DX (Fig. 1D). Although young breast cancer patients (35-45 years) utilized Oncotype DX at the same rate as middle-aged patients in the first few years after the introduction of Oncotype DX (20042007), their usage in the latter part of the study period was almost identical to older patients with breast cancer (Fig. 1D).

\section{Oncotype DX testing is associated with decreased usage of chemotherapy}

An important application of the Oncotype DX assay is to aid physicians in deciding whether adjuvant chemotherapy would likely benefit a patient with breast cancer. We therefore investigated the relationship between Oncotype DX testing and chemotherapy usage. As shown, from 2004 to 2015, the use of Oncotype DX in $\mathrm{ER}+$ breast cancer patients increased steadily from $1.5 \%$ to $34 \%$ (Fig. 2A). Meanwhile, the rate of chemotherapy usage continuously decreased from 42 to $36 \%$ (Fig. 2A). This trend is likely related to the decrease in
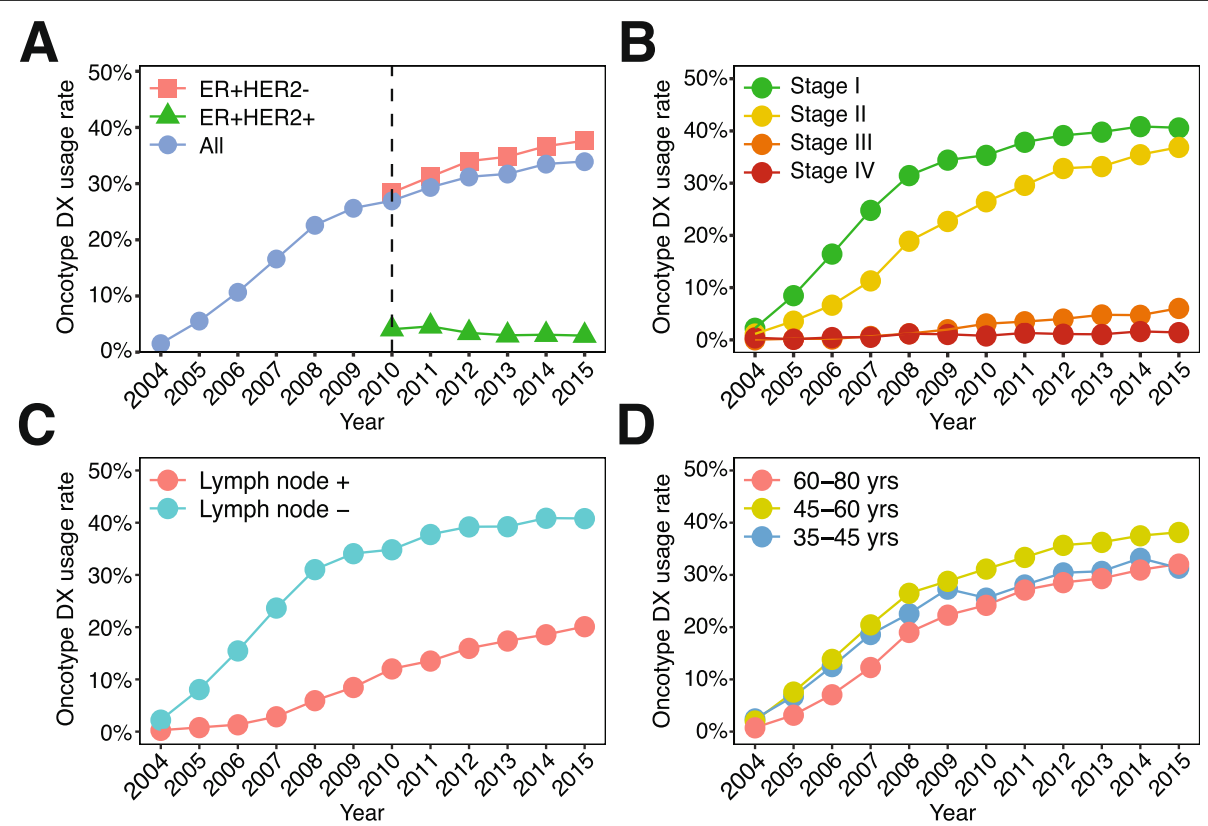

Fig. 1 Clinical characteristics of Oncotype DX users between 2004 and 2015. A Oncotype DX usage rate among patients with ER+HER2-, ER+HER2+, and all breast cancer subtypes. B Oncotype DX usage rate among the four stages of breast cancer. C Oncotype DX usage rate between patients with lymph node-positive and negative breast cancer. D Oncotype DX usage rate among different patient age groups. Usage rates were calculated based on the total number of patients within each indicated patient subgroup 

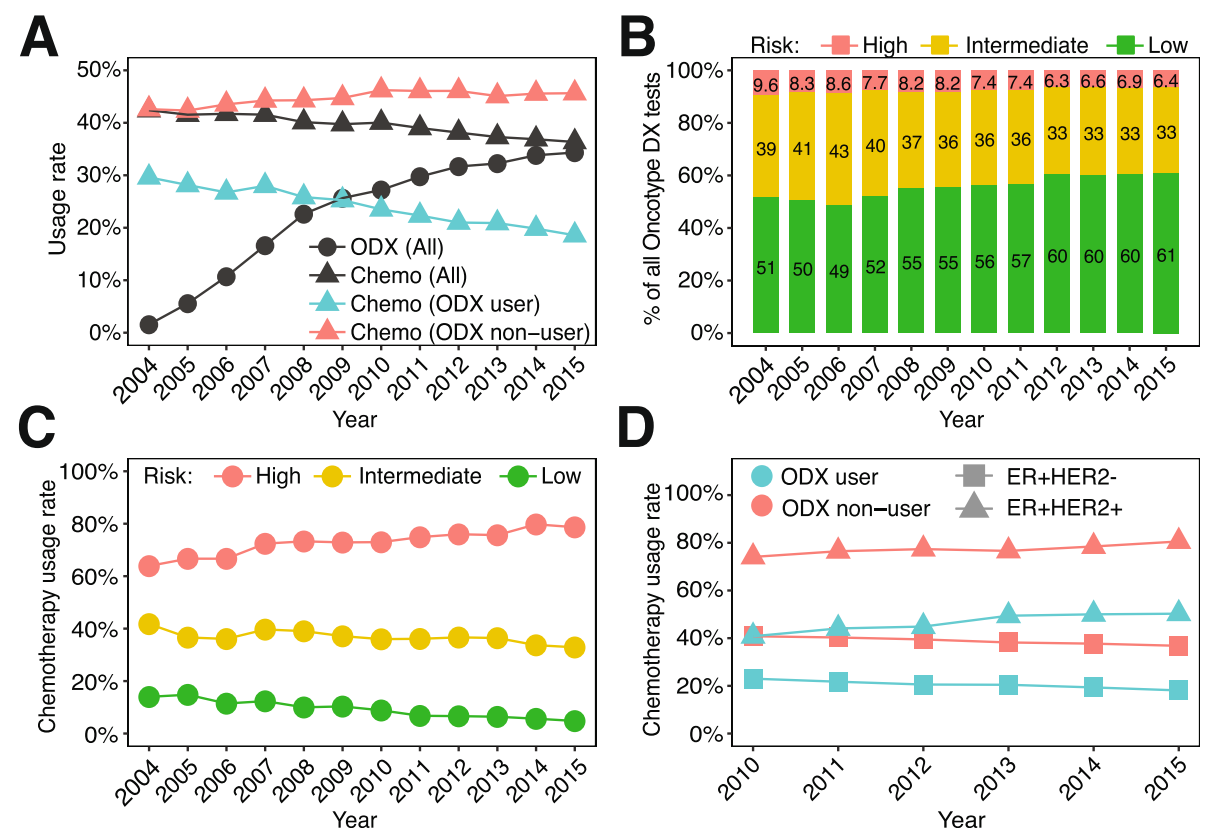

Fig. 2 Usage of chemotherapy in Oncotype DX users between 2004 and 2015. A Oncotype DX usage rates among patients undergoing chemotherapy (triangles) and overall Oncotype DX usage rate (circles). B Percentage of risk groups (high-, intermediate-, and low-risk) classified by Oncotype DX test results. C Percentage of chemotherapy usage between high-, intermediate-, and low-risk Oncotype DX groups. D Chemotherapy usage among patients with ER+HER2 - and ER+HER2+ breast cancer, stratified by Oncotype DX usage. For A, C, and D, usage rates were calculated based on the total number of patients within each indicated patient subgroup

chemotherapy usage in patients who underwent Oncotype DX testing; the rate of chemotherapy usage continuously decreased in Oncotype DX users, but continuously increased in non-users from 2004 to 2015 (Fig. 2A).

With more ER+ breast cancer patients utilizing the Oncotype DX assay, an overall decrease in the percentage of high-risk patients (from 9.6\% in 2004 to $6.3 \%$ in 2015) and an increase in the percentage of low-risk patients (from $51 \%$ in 2004 to $61 \%$ in 2015) were observed (Fig. 2B). These percentages are similar to previously reported percentages [29-31]. When examining the usage of chemotherapy in the three risk groups, we observed that the chemotherapy rate continuously increased in the high-risk group, but slowly decreased year by year in the intermediate- and low-risk groups (Fig. 2C). This is consistent with the guidelines of Oncotype DX, which recommend adjuvant chemotherapy for ER+ breast cancer patients with high recurrence risk and no adjuvant chemotherapy for patients with low risk of recurrence [14]. Chemotherapy was more frequently used to treat patients with ER+HER2+ compared to ER+HER2- breast cancer (Fig. 2D). In both of these breast cancer subtypes, Oncotype DX users showed a lower rate of chemotherapy usage compared to non-users.

\section{Oncotype DX users tend to have better prognosis}

To examine the potential benefit from Oncotype DX test results, we compared the survival time between Oncotype DX users and non-users. Univariate Cox regression analysis indicated that users tended to have significantly longer BCSS compared to non-users in all years from 2004 to 2015 (Fig. 3A). Relative to non-users, users showed HRs around 0.2, indicating an $80 \%$ lower risk of breast cancer-specific death. According to the guidelines, Oncotype DX tests are recommended for ER+ patients with lower tumor stages and negative lymph node status [14], which are clinical characteristics that are known to be associated with good prognosis. Thus, multivariable Cox regression models were used to adjust for tumor stage and lymph node status, as well as for patients' age. As shown, after adjustment, Oncotype DX test users still demonstrated significantly longer survival times compared to nonusers (Fig. 3A). The overall benefit from genomic testing continuously increased from 2006 to 2015 with hazard ratios trending down over this time period.

To obtain a more detailed association between Oncotype DX usage, patient prognosis, and clinical variables, we next stratified patients based on tumor stage. A significant benefit from the Oncotype DX test was observed for both late stage (2009-2015) and early-stage (in all years) in ER+ breast cancer patients (Fig. 3B). Without 
A
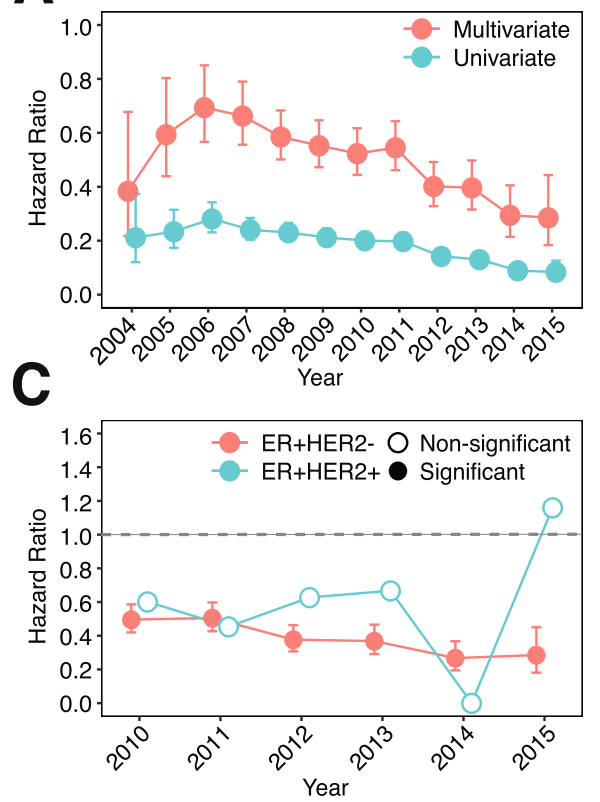

B
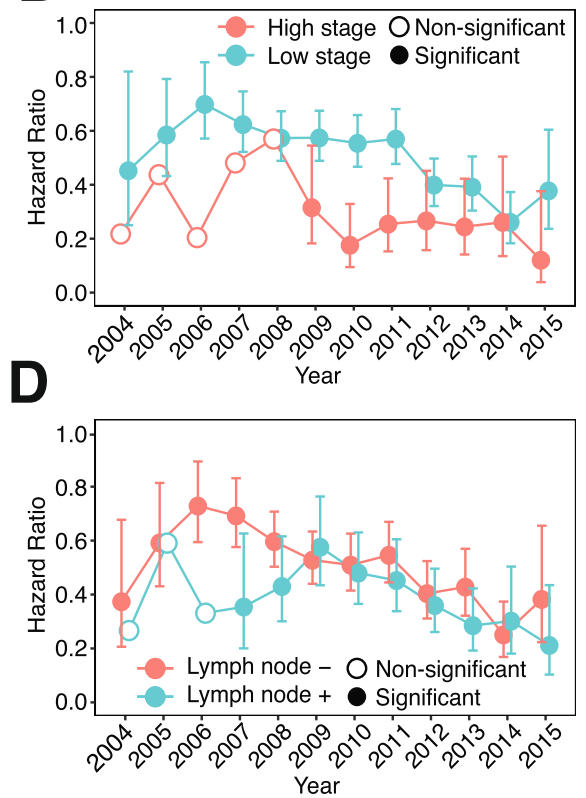

Fig. 3 Use of Oncotype DX improves survival. A Hazard ratio of Oncotype DX usage between 2004 and 2015 from univariate and multivariable Cox models. Multivariable Cox models were adjusted for tumor stage, tumor grade, lymph node status, breast cancer subtype (ER+HER2- or ER+HER2+), and patients' age. B Hazard ratios of Oncotype DX usage stratified by tumor stage between 2004 and 2015 from univariate Cox regression models. C Hazard ratio of Oncotype DX usage stratified by subtypes between 2010 and 2015 from univariate Cox regression models. D Hazard ratio of Oncotype DX usage stratified by lymph node status between 2004 and 2015 from univariate Cox regression models. P values below 0.05 were considered significant

considering other clinical variables, patients with latestage disease seemed to benefit more from Oncotype DX testing, showing lower hazard ratios compared to early-stage disease (Fig. 3B). The prognosis of patients with low and high grade tumors was identical, showing comparable hazard ratios for almost all evaluated years (Additional file 1 - Figure 1B). For patients with ER+ HER2 - breast cancer, Oncotype DX users showed consistent benefit with significant hazard ratios in all evaluated years (Fig. 3C). Patients with ER+HER2+ breast cancer seemed to also benefit from Oncotype DX testing, but significantly longer BCSS was not observed compared to non-users in all years (Fig. 3C). In addition, both lymph node-positive (in all years) and negative (2007-2015) patients benefited from the Oncotype DX testing (Fig. 3D). Taking together, our results indicate that patients with ER+HER2- breast cancer demonstrated significant benefit from the Oncotype DX test regardless of tumor stage and lymph node status.

\section{Oncotype DX score is predictive of patient survival}

To confirm the prognostic value of the Oncotype DX test, we evaluated BCSS of ER+ patients in the three risk-recurrence groups. As shown, patients in the highrisk group had significantly worse BCSS compared to the intermediate- and the low-risk groups; the intermediate-risk group had significantly worse BCSS than the low-risk group (Fig. 4A, Additional file 1 Figure 2A). Multivariable Cox regression analysis adjusting for age, stage, grade, lymph node status, and breast cancer subtype also showed consistently worse BCSS for high-risk patients in all years except for 2004 (presumably due to small patient number) and 2015 (presumably due to short follow-up time) with HR ranging from 3.25 to 10.36 compared to low-risk patients (Fig. 4B). For intermediate-risk patients, significantly worse BCSS was observed in the majority of evaluated years but with much lower HRs (Fig. 4B). Overall, the high- and intermediate-risk patients showed approximately sixfold and twofold higher risk of decreased BCSS, respectively, compared to patients in the low-risk group. When combining all years, an identical observation was made; both the intermediate and high-risk patients had significantly shorter BCSS compared to low-risk patients in a multivariable Cox regression model (Additional file 2 - Table 1). Similar findings were obtained using OS instead of BCSS, but the degree of significance tended to be lower (Fig. $4 \mathrm{C}$, D, Additional file 1 - Figure 2B, Additional file 2 - Table 2). 
A
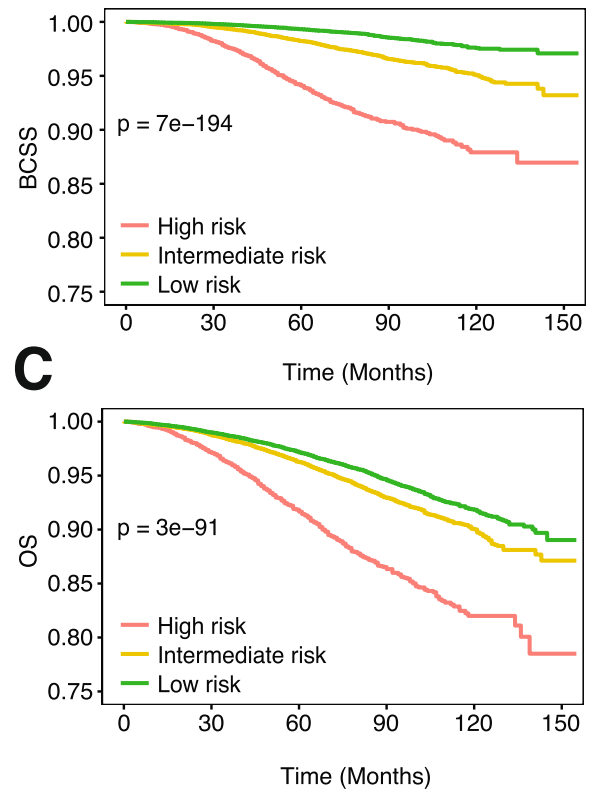

B
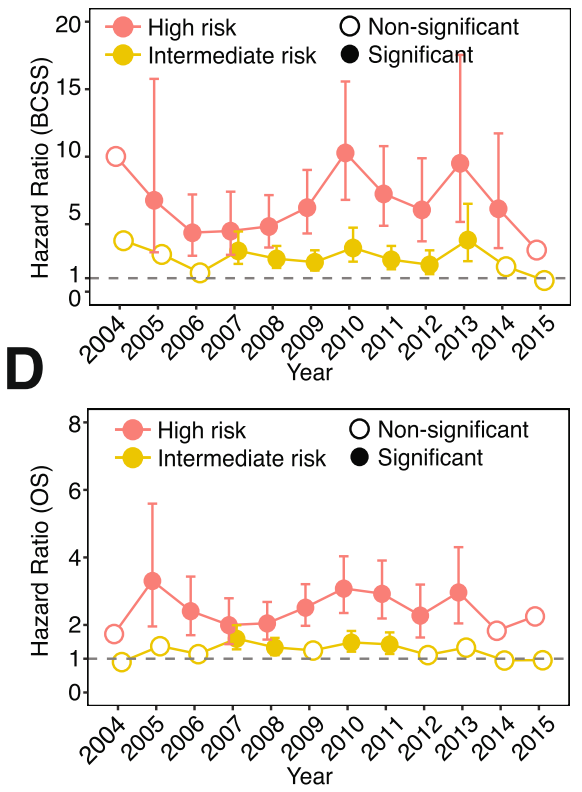

Fig. 4 Oncotype DX score is predictive of patient's survival. A Survival plot of 3 risk groups classified by Oncotype DX (BCSS). B Hazard ratio of high and intermediate-risk groups using low-risk as baseline (BCSS). Multivariable Cox models were adjusted for tumor stage, lymph node status, breast cancer subtype (ER+HER2 - or ER+HER2+), and patients' age. C Survival plot of 3 risk groups classified by Oncotype DX (OS). D Hazard ratio of high and intermediate-risk groups using low-risk as baseline (OS). Multivariable Cox models were adjusted for tumor stage, lymph node status, breast cancer subtype (ER+HER2- or ER+HER2+), and patients' age. $P$ values below 0.05 were considered significant

\section{Chemotherapy improves the prognosis of high-risk patients identified by the Oncotype DX test}

Oncotype DX has been used to decide the use of adjuvant chemotherapy in ER+ breast cancer. We thus performed multivariable Cox regression analysis to examine whether chemotherapy improved the prognosis of patients. As shown in Table 2, we found that high-risk patients benefited from chemotherapy with the risk of overall death decreasing by $40 \%$ after chemotherapy use as compared to high-risk patients with no/unknown chemotherapy use $(\mathrm{HR}=0.60, p=7.8 \mathrm{E}-5$, Table 2). Patients with intermediate-risk also benefited from chemotherapy use compared to intermediate-risk patients with no/unknown chemotherapy use $(\mathrm{HR}=0.74, p=2.8 \mathrm{E}-4$, Table 2). In contrast, chemotherapy was not associated with prolonged OS for patients with low-risk $(p>0.05$, Table 2).

When we restricted our analysis to ER+HER2- breast cancer, the results similarly indicated a protective effect of chemotherapy in the high-risk ( $\mathrm{HR}=0.62, p=8.0 \mathrm{E}$ -4 , Table 2) and intermediate-risk group ( $\mathrm{HR}=0.79, p$ $=0.01$, Table 2). Again, no association between chemotherapy and OS was observed in the low-risk group. For patients with ER+HER2+ breast cancer, identical trends were observed (Table 2). Similar findings were also observed using BCSS although no significant relationship was observed between prolonged BCSS and chemotherapy usage in the intermediate-risk group (Additional file 2 - Table 1).

Interestingly, radiation therapy was protective in patients with intermediate or low Oncotype DX scores but not in patients with high Oncotype DX scores (Table 2). In intermediate-risk patients, an HR of $0.72(p=1.5 \mathrm{E}-5)$ suggested that patients who underwent radiation therapy had longer OS compared to patients who did not undergo radiation therapy. Similarly, an HR of 0.76 ( $p=$ $2.0 \mathrm{E}-4)$ in low-risk patients suggested prolonged OS after the use of radiotherapy (Table 2). A similar observation was made when using BCSS although the association in low-risk patients did not reach statistical significance (Additional file 2 - Table 1).

\section{Discussion}

In this study, we investigated the temporal trends of Oncotype DX usage the first decade after the introduction of the Oncotype DX test in the USA. We found that Oncotype DX was mostly used by ER+ breast cancer patients with negative lymph node status, who were between 45 and 60 years of age. Compared to non-users, Oncotype DX users tended to have significantly longer breast cancer-specific and overall survival after adjustment of clinical factors. We observed an inverse trend between Oncotype DX usage and chemotherapy, which was mainly driven by the decreased chemotherapy rate 
Table 2 Chemotherapy increases overall survival in high-risk score patients.

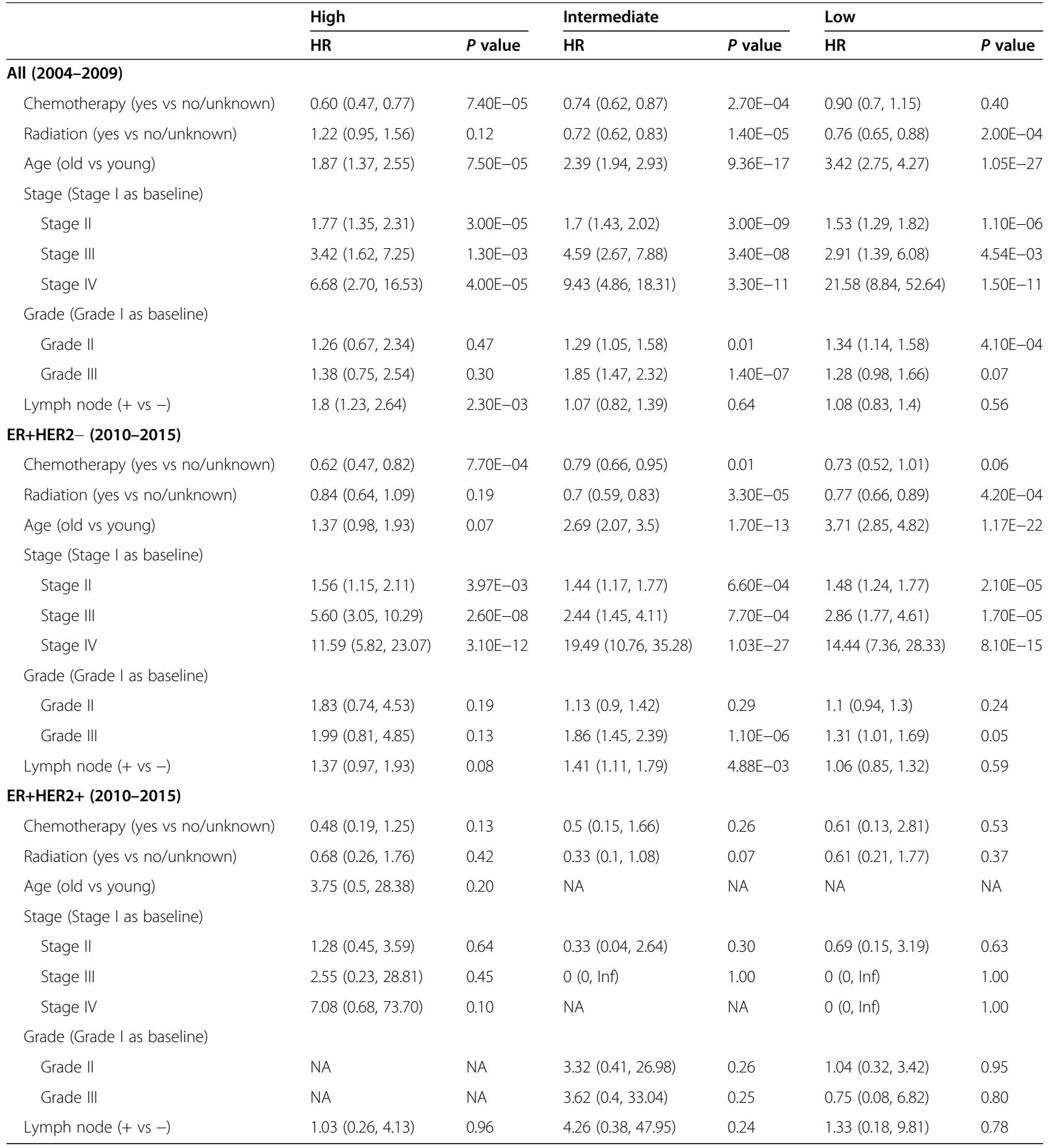

in patients with low recurrence-risk test results. Survival analyses validated that high-risk patients had significantly worse survival than patients in other risk groups. Moreover, our results indicated that only high-risk but not intermediate or low-risk patients benefit from chemotherapy. These results provide useful information to refine the guidelines for directing Oncotype DX tests.
The current NCCN guidelines for eligible patients include ER+, early-stage (T1 or T2), lymph node-negative (pN0) breast cancer patients [14]. We observed that the majority of performed Oncotype DX tests followed these guidelines, but also noted a slight increase in the use of Oncotype DX among later breast cancer stages and lymph node-positive breast cancer. Based on our survival 
analysis, both lymph node-negative and positive patients had improved survival after undergoing Oncotype DX testing (Fig. 3). A number of studies have indeed suggested the clinical benefit of Oncotype DX test results in treatment decision-making for lymph node-positive breast cancer [32-34]. Indeed, early results from the large RxPONDER (NCT01272037) trial [13] have indicated that ER+HER2- patients with early-stage, lymph node-positive breast cancer with an Oncotype Dx score $\leq 25$ can safely forgo adjuvant chemotherapy.

We have shown that Oncotype DX testing is associated with decreased adjuvant chemotherapy usage and increased survival. Even after adjusting for several clinical variables, including tumor stage and lymph node status, this association between Oncotype DX usage and survival was still significant and suggested an $40 \%$ lower risk of breast cancer-specific death upon Oncotype DX usage. While the current study did not address changes in treatment recommendations by physicians/patients after obtaining Oncotype DX results, we hypothesize that altered decision-making underlies this survival benefit. It has been reported that treatment recommendations are reconsidered in $\sim 30-45 \%$ of patients after Oncotype DX test results became available [16, 29, 30, 35-38]. In the majority of these cases, adjuvant chemotherapy was withheld after reconsideration. Consistently, BCSS was significantly shorter in low risk patients treated with chemotherapy (Additional file 2 - Table 1), suggesting a hazardous role for chemotherapy in this subset of patients.

In this cohort, $6.8 \%$ of ER+ breast cancers had HER2+ breast cancer. Oncotype DX testing is not recommended for ER+HER2+ breast cancer [14] due to the availability of HER2-specific therapies for this group of patients. However, approximately $4.7 \%$ of ER+HER2+ patients underwent Oncotype DX testing. These patients are a special subset of all ER+HER2+ patients who were HER2+ according to SEER standards but were HER2based on the Oncotype DX assay. This group of patients had lower rates of chemotherapy usage and did not obtain significant benefits from Oncotype DX testing in terms of OS and BCSS. Consequently, the Oncotype DX test might not be as beneficial to this subset of ER+ HER2+ breast cancer compared to ER+HER2- breast cancer.

Although our study provides valuable insights into the use of Oncotype DX testing in breast cancer, some limitations should be noted. First, it is likely that additional patients' characteristics are associated with Oncotype DX usage besides the variables that we evaluated in this study. For example, marital and insurance status have previously been associated with Oncotype DX usage [24]. Second, the usage of chemotherapy and radiation therapy tends to be underreported in the SEER cohort. For example, a number of patients had "no/unknown" chemotherapy and radiation status, which means that a subset of these patients likely did receive either of these therapies but was classified as "unknown". This might have affected some of our results. Lastly, our results pertaining to ER+HER2+ breast cancer patients need to be interpreted with caution since this group of patients is likely to represent a subset of ER+HER2+ patients.

\section{Conclusion}

We have provided a comprehensive temporal overview of the use of Oncotype DX in breast cancer patients in the first decade after Oncotype DX was introduced. Our results suggest that the use of Oncotype DX is steadily increasing in ER+HER2- breast cancer. The provided risk scores provide valuable information for patient prognosis and help guide treatment decisions.

\section{Abbreviations}

ASCO: American Society of Clinical Oncology; BCSS: Breast cancer-specific survival; ER: Estrogen receptor; HR: Hazard ratio; HER2: Human epidermal growth factor receptor 2; NCCN: National Comprehensive Cancer Network; OS: Overall survival; pN+: Lymph node-positive; pNO: Lymph node-negative; PR: Progesterone receptor; SEER: Surveillance, Epidemiology, and End Results

\section{Supplementary Information}

The online version contains supplementary material available at https://doi. org/10.1186/s13058-021-01453-4.

Additional file 1: Supplementary Figures. Two supplementary figures.

Additional file 2: Supplementary Tables. Two supplementary tables.

\section{Acknowledgements}

We would like to thank all members of the Cheng lab for their valuable discussions and critical feedback.

\section{Authors' contributions}

C.C. conceived the project. C.Y.T. obtained the data. E.S., B.Z., M.S., and C.C. performed computational analyses. E.S., B.Z., M.S., L.Z., and C.C. wrote the manuscript. E.S., B.Z, M.S., C.Y.T, L.Z., and C.C. interpreted the results. C.C. supervised the project. All authors critically reviewed the content. All authors read and approved the final manuscript.

\section{Funding}

This work is supported by the Cancer Prevention Research Institute of Texas (CPRIT; RR180061, to C. Cheng) and the NCl of the NIH (1R21CA227996, to C. Cheng), and the T32 training grant of the NIH (T32 Al007363, to E.

Schaafsma). C. Cheng is a CPRIT Scholar in Cancer Research.

Availability of data and materials

All data utilized in this study are publicly available.

\section{Declarations}

Ethics approval and consent to participate N/A

Consent for publication

N/A 


\section{Competing interests}

The authors declare that they have no potential competing interests.

\begin{abstract}
Author details
'Department of Molecular and Systems Biology, Dartmouth College, Hanover, NH 03755, USA. ${ }^{2}$ Department of Chemical and Biomolecular Engineering, Rice University, Houston, TX 77030, USA. ${ }^{3}$ Faculty of Medical Sciences, University of Groningen, Groningen, The Netherlands. ${ }^{4}$ Department of Medicine, Baylor College of Medicine, Houston, TX 77030, USA. ${ }^{5}$ Department of Biological Sciences, Rutgers University Newark, Newark, NJ, USA. ${ }^{6}$ Department of Pathology, Princeton Medical Center, Plainsboro, NJ, USA. ${ }^{7}$ Dan L Duncan Comprehensive Cancer Center, Baylor College of Medicine, Houston, TX 77030, USA. ${ }^{8}$ Department of Biomedical Data Science, Geisel School of Medicine at Dartmouth, Lebanon, NH 03756, USA. 'The Institute for Clinical and Translational Research, Baylor College of Medicine, Houston, TX 77030, USA.
\end{abstract}

Received: 22 December 2020 Accepted: 8 July 2021

Published online: 17 July 2021

\section{References}

1. Ferlay J, Soerjomataram I, Dikshit R, Eser S, Mathers C, Rebelo M, et al. Cancer incidence and mortality worldwide: sources, methods and major patterns in GLOBOCAN 2012. Int J Cancer. 2015;136(5):E359-86. https://doi. org/10.1002/ijc.29210.

2. Momenimovahed $Z$, Salehiniya $H$. Epidemiological characteristics of and risk factors for breast cancer in the world. Breast Cancer. 2019;11:151-64.

3. Harbeck N, Penault-Llorca F, Cortes J, Gnant M, Houssami N, Poortmans P. et al. Breast cancer. Nat Rev Dis Prim. 2019;5:1-31.

4. Howlader N, Altekruse SF, Li Cl, Chen WW, Clarke CA, Ries LAG, et al. US Incidence of Breast Cancer Subtypes Defined by Joint Hormone Receptor and HER2 Status. J Natl Cancer Inst. 2014;106(5). https://doi.org/10.1093/jnci/ dju055.

5. Female Breast Cancer Subtypes - Cancer Stat Facts. SEER. Available from: https://seer.cancer.gov/statfacts/html/breast-subtypes.html

6. Fisher B, Costantino J, Redmond C, Poisson R, Bowman D, Couture J, et al. A randomized clinical trial evaluating tamoxifen in the treatment of patients with node-negative breast cancer who have estrogen-receptor-positive tumors. N Engl J Med. 1989;320(8):479-84. https://doi.org/10.1056/NEJM1 98902233200802.

7. Fisher B, Jeong J-H, Bryant J, Anderson S, Dignam J, Fisher ER, et al. Treatment of lymph-node-negative, oestrogen-receptor-positive breast cancer: long-term findings from National Surgical Adjuvant Breast and Bowel Project randomised clinical trials. Lancet. 2004;364(9437):858-68. https://doi.org/10.1016/S0140-6736(04)16981-X.

8. Early Breast Cancer Trialists' Collaborative Group (EBCTCG). Comparisons between different polychemotherapy regimens for early breast cancer: meta-analyses of long-term outcome among 100000 women in 123 randomised trials. Lancet. 2012;379:432-44.

9. Paik S, Shak S, Tang G, Kim C, Baker J, Cronin M, et al. A Multigene Assay to Predict Recurrence of Tamoxifen-Treated, Node-Negative Breast Cancer. N Engl J Med. 2004;351(27):2817-26. https://doi.org/10.1056/NEJMoa041588.

10. Habel LA, Shak S, Jacobs MK, Capra A, Alexander C, Pho M, et al. A population-based study of tumor gene expression and risk of breast cancer death among lymph node-negative patients. Breast Cancer Res. 2006;8(3): R25. https://doi.org/10.1186/bcr1412.

11. Ross JS, Hatzis C, Symmans WF, Pusztai L, Hortobágyi GN. Commercialized multigene predictors of clinical outcome for breast cancer. Oncologist. 2008;13(5):477-93. https://doi.org/10.1634/theoncologist.2007-0248.

12. Harris $L$, Fritsche $H$, Mennel $R$, Norton $L$, Ravdin P, Taube $S$, et al. American Society of Clinical Oncology 2007 update of recommendations for the use of tumor markers in breast cancer. J Clin Oncol. 2007;25(33):5287-312. https://doi.org/10.1200/JCO.2007.14.2364.

13. Ramsey SD, Barlow WE, Gonzalez-Angulo AM, Tunis S, Baker L, Crowley J, et al. Integrating comparative effectiveness design elements and endpoints into a phase III, randomized clinical trial (SWOG S1007) evaluating oncotypeDX-guided management for women with breast cancer involving lymph nodes. Contemp Clin Trials. 2013;34(1):1-9. https://doi.org/10.1016/j. cct.2012.09.003.

14. National Comprehensive Cancer Network. NCCN guidelines, version 6. 2020; 2020.
15. Partin JF, Mamounas EP. Impact of the 21-gene recurrence score assay compared with standard clinicopathologic guidelines in adjuvant therapy selection for node-negative, estrogen receptor-positive breast cancer. Ann Surg Oncol. 2011;18(12):3399-406. https://doi.org/10.1245/s1 0434-011-1698-z.

16. Ademuyiwa FO, Miller A, O'Connor T, Edge SB, Thorat MA, Sledge GW, et al. The effects of oncotype DX recurrence scores on chemotherapy utilization in a multi-institutional breast cancer cohort. Breast Cancer Res Treat. 2011; 126(3):797-802. https://doi.org/10.1007/s10549-010-1329-6.

17. Kapadia S, Gudiwada SP, Kaji AH, Chlebowski RT, Venegas R, Ozao-Choy J, et al. Can Oncotype DX testing be omitted in invasive breast cancer patients with clinicopathologic factors predicting very high pretest probability of a concordant result? Breast J. 2020;26(11):2199-202.

18. Losk K, Freedman RA, Laws A, Kantor O, Mittendorf EA, Tan-Wasielewski Z, et al. Oncotype DX testing in node-positive breast cancer strongly impacts chemotherapy use at a comprehensive cancer center. Breast Cancer Res Treat. 2020.

19. Tzeng JP, Mayer D, Richman AR, Lipkus I, Han PK, Valle CG, et al. Women's experiences with genomic testing for breast cancer recurrence risk. Cancer. 2010;116(8):1992-2000. https://doi.org/10.1002/cncr.24990.

20. Guth AA, Fineberg S, Fei K, Franco R, Bickell N. Utilization of Oncotype DX to predict chemotherapy use in an inner-city population. JCO Am Soc Clin Oncol. 2011;29:e11131.

21. Goodwin M, Hughes J, Diego M, Frazier T. Impact of OncoType DXTM Recurrence Score and Tumor Size on Making Chemotherapy Decisions in Breast Cancer Patients. Cancer Res. 2009;69:6039.

22. Gianni L, Zambetti M, Clark K, Baker J, Cronin M, Wu J, et al. Gene expression profiles in paraffin-embedded core biopsy tissue predict response to chemotherapy in women with locally advanced breast cancer. J Clin Oncol. 2005;23(29):7265-77. https://doi.org/10.1200/JCO.2005.02.0818.

23. Sparano JA, Gray RJ, Makower DF, Pritchard Kl, Albain KS, Hayes DF, et al. Adjuvant Chemotherapy Guided by a 21-Gene Expression Assay in Breast Cancer. N Engl J Med. 2018;379:111-21.

24. Roberts MC, Miller DP, Shak S, Petkov VI. Breast cancer-specific survival in patients with lymph node-positive hormone receptor-positive invasive breast cancer and Oncotype DX Recurrence Score results in the SEER database. Breast Cancer Res Treat. 2017;163(2):303-10. https://doi.org/10.1 007/s10549-017-4162-3.

25. McVeigh TP, Hughes LM, Miller N, Sheehan M, Keane M, Sweeney KJ, et al. The impact of Oncotype DX testing on breast cancer management and chemotherapy prescribing patterns in a tertiary referral centre. Eur J Cancer. 2014;50(16):2763-70. https://doi.org/10.1016/j.ejca.2014.08.002.

26. Petkov VI, Miller DP, Howlader N, Gliner N, Howe W, Schussler N, et al. Breast-cancer-specific mortality in patients treated based on the 21-gene assay: a SEER population-based study. NPJ Breast Cancer. 2016;2(1):16017. https://doi.org/10.1038/npjbcancer.2016.17.

27. Surveillance, Epidemiology, and End Results (SEER) Program (www.seer.ca ncer.gov): Oncotype DX Database (2004-2015), National Cancer Institute, DCCPS, Surveillance Research Program. 2019.

28. Lund MJ, Mosunjac M, Davis KM, Gabram-Mendola S, Rizzo M, Bumpers HL, et al. 21-Gene recurrence scores: racial differences in testing, scores, treatment, and outcome. Cancer. 2012;118(3):788-96. https://doi.org/10.1 002/cncr.26180.

29. Green N, Al-Allak A, Fowler C. Benefits of introduction of Oncotype DX testing. Ann R Coll Surg Engl. 2019;101(1):55-9. https://doi.org/10.1308/rcsa $\mathrm{nn} .2018 .0173$

30. Carlson JJ, Roth JA. The impact of the Oncotype Dx breast cancer assay in clinical practice: a systematic review and meta-analysis. Breast Cancer Res Treat. 2013;141(1):13-22. https://doi.org/10.1007/s10549-013-2666-z.

31. Joh JE, Esposito NN, Kiluk JV, Laronga C, Lee MC, Loftus L, et al. The effect of Oncotype DX recurrence score on treatment recommendations for patients with estrogen receptor-positive early stage breast cancer and correlation with estimation of recurrence risk by breast cancer specialists. Oncologist. 2011;16(11):1520-6. https://doi.org/10.1634/theoncologist.20110045

32. Goldstein $\sqcup$, Gray R, Badve S, Childs BH, Yoshizawa C, Rowley S, et al. Prognostic utility of the 21-gene assay in hormone receptor-positive operable breast cancer compared with classical clinicopathologic features. J Clin Oncol. 2008;26(25):4063-71. https://doi.org/10.1200/JCO.2007.14.4501.

33. Albain KS, Barlow WE, Shak S, Hortobagyi GN, Livingston RB, Yeh I-T, et al. Prognostic and predictive value of the 21 -gene recurrence score assay in 
postmenopausal women with node-positive, oestrogen-receptor-positive breast cancer on chemotherapy: a retrospective analysis of a randomised trial. Lancet Oncol. 2010;11(1):55-65. https://doi.org/10.1016/S1470-204 5(09)70314-6.

34. Dowsett M, Cuzick J, Wale C, Forbes J, Mallon EA, Salter J, et al. Prediction of risk of distant recurrence using the 21 -gene recurrence score in nodenegative and node-positive postmenopausal patients with breast cancer treated with anastrozole or tamoxifen: a TransATAC study. J Clin Oncol. 2010;28(11):1829-34. https://doi.org/10.1200/JCO.2009.24.4798.

35. Henry LR, Stojadinovic A, Swain SM, Prindiville S, Cordes R, Soballe PW. The influence of a gene expression profile on breast cancer decisions. J Surg Oncol. 2009;99(6):319-23. https://doi.org/10.1002/jso.21244.

36. Lin C-Y, Mooney K, Choy W, Yang S-R, Barry-Holson K, Horst K, et al. Will oncotype DX DCIS testing guide therapy? A single-institution correlation of oncotype DX DCIS results with histopathologic findings and clinical management decisions. Mod Pathol. 2018;31(4):562-8. https://doi.org/10.103 8/modpathol.2017.172.

37. Rutter $C E$, Yao X, Mancini BR, Aminawung JA, Chagpar AB, Saglam O, et al. Influence of a 21-Gene Recurrence Score Assay on Chemotherapy Delivery in Breast Cancer. Clin Breast Cancer. 2016;16(1):59-62. https://doi.org/10.101 6/j.clbc.2015.09.008.

38. Asad J, Jacobson AF, Estabrook A, Smith SR, Boolbol SK, Feldman SM, et al. Does oncotype DX recurrence score affect the management of patients with early-stage breast cancer? Am J Surg. 2008;196(4):527-9. https://doi. org/10.1016/j.amjsurg.2008.06.021.

\section{Publisher's Note}

Springer Nature remains neutral with regard to jurisdictional claims in published maps and institutional affiliations.

Ready to submit your research? Choose BMC and benefit from:

- fast, convenient online submission

- thorough peer review by experienced researchers in your field

- rapid publication on acceptance

- support for research data, including large and complex data types

- gold Open Access which fosters wider collaboration and increased citations

- maximum visibility for your research: over $100 \mathrm{M}$ website views per year

At BMC, research is always in progress.

Learn more biomedcentral.com/submissions 